\title{
Laparoscopy-Assisted Transgastric ERCP: A Challenging Technique for Biliary Clearance Post Roux-en-Y Gastric Bypass
}

\author{
Hitendra Kumar Garg ${ }^{1}$ Pankaj Singh ${ }^{1} \quad$ Atul N. C. Peters ${ }^{2}$ Shalabh Agarwal ${ }^{2}$ Yogesh Gautam² \\ Dhruv Kant Mishra'
}

${ }^{1}$ Department of Gastroenterology and Hepatology, Indraprastha Apollo Hospital, New Delhi, India

2Department of Bariatric, Minimal Access and General Surgery, Max Smart Super Speciality Hospital, New Delhi, India

\begin{abstract}
Address for correspondence Pankaj Singh, MD, DM, Department of Gastroenterology and Transplant Hepatology, Indraprastha Apollo Hospital, New Delhi 110076, India (e-mail: pankajdoc06@gmail.com).
\end{abstract}

J Digest Endosc 2021;12:107-111.

\begin{abstract}
Biliary complications after Roux-en-Y gastric bypass (RYGB) are not uncommon. Managing choledocholithiasis in this subset of patients is challenging as conventional endoscopic retrograde cholangiopancreatography (ERCP) is not possible due to lack

Keywords

- Roux-en-Y gastric bypass

- RYGB

- laparoscopy-assisted transgastric endoscopic retrograde cholangiopancreatography

- LA-ERCP of peroral access to the second part of duodenum. Enteroscopic approach, however, theoretically feasible is difficult and cumbersome, often resulting in failed procedure. Small series has reported higher success with a novel technique of laparoscopic transgastric access for ERCP. Laparoscopy-assisted ERCP (LA-ERCP) is now increasingly being recognized as a safe and reliable technique for biliary clearance with a low complication rate, making it a preferred approach for managing biliopancreatic disease in these groups of patients. Here we report a case of a young patient with gallstone disease and choledocholithiasis presenting with obstructive jaundice post RYGB who was successfully managed by LA-ERCP followed by laparoscopic cholecystectomy in the same session.
\end{abstract}

\section{Introduction}

The prevalence of obesity is rising worldwide necessitating increasing need for bariatric surgery. Of different surgical modalities Roux-en-Y gastric bypass (RYGB) is one of the most commonly done procedure. Rapid weight loss in these patients renders increased production of lithogenic bile favoring more gallstone diseases. ${ }^{1}$ Cholelithiasis is common and is seen in around $40 \%$ patients within 6 months of surgery. ${ }^{2}$ However, there is some recommendation for concomitant cholecystectomy at the time of bariatric surgery but no definite international guideline or consensus on this topic. ${ }^{3}$ RYGB alters upper gastrointestinal anatomy in such a way that any endoscopic biliary intervention is technically challenging
(Fig. 1A). Conventional transoral endoscopic retrograde cholangiopancreatography (ERCP) is not feasible; however, balloon enteroscopy-assisted ERCP (BEA-ERCP) can be done, but often with low rate of successful biliary cannulation. An alternative approach is laparoscopy-assisted transgastric endoscopic retrograde cholangiopancreatography (LA-ERCP) in which laparoscopic access to the remnant stomach is used as a conduit through which side view endoscope can be passed into the duodenum and approach papilla in the conventional manner. Here we report our first experience of this technically challenging procedure of LA-ERCP post RYGB with successful biliary clearance and concomitant cholecystectomy. published online

September 1, 2021
DOI https://doi.org/

$10.1055 / \mathrm{s}-0041-1728221$ ISSN 0976-5042 (c) 2021. Society of Gastrointestinal Endoscopy of India.

This is an open access article published by Thieme under the terms of the Creative Commons Attribution-NonDerivative-NonCommercial-License, permitting copying and reproduction so long as the original work is given appropriate credit. Contents may not be used for commercial purposes, or adapted, remixed, transformed or built upon. (https://creativecommons.org/licenses/by-nc-nd/4.0/). Thieme Medical and Scientific Publishers Pvt. Ltd. A-12, 2nd Floor, Sector 2, Noida-201301 UP, India 

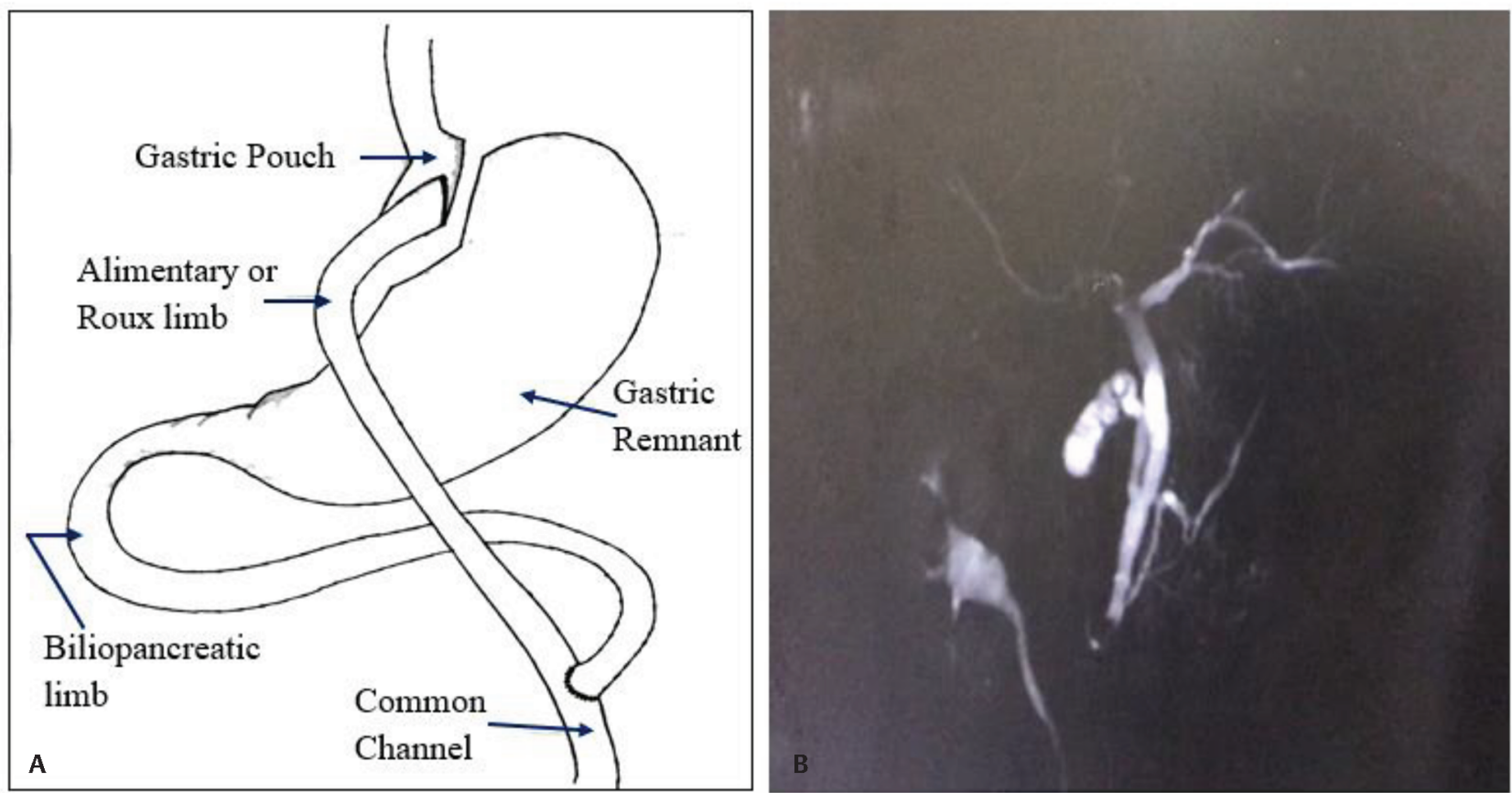

Fig. 1 (A) Schematic representative of the anatomy of the upper gastrointestinal tract following Roux-en-Y gastric bypass; (B) Magnetic resonance cholangiopancreatography image showing cholelithiasis with prominent common bile duct with calculi at lower end.

\section{Case Report}

A 40-year-old healthy Indian male with a history of bariatric RYGB 2 years back presented with recurrent upper abdominal pain for 2 months and new onset jaundice for 1 week. Blood tests revealed deranged liver function test with total bilirubin of $2.4 \mathrm{mg} / \mathrm{dL}$, elevated liver enzymes: serum aspartate aminotransferase of $65 \mathrm{U} / \mathrm{L}$, alanine aminotransferase of $85 \mathrm{U} / \mathrm{L}$, alkaline phosphatase, and gamma-glutamyl transpeptidase value of 329 and 190 U/L, respectively, suggestive predominantly of cholestatic pattern. His complete blood count and kidney functions were normal. Ultrasound showed multiple gallstones with almost normal sized common bile duct (CBD) $\sim 5 \mathrm{~mm}$. Magnetic resonance cholangiopancreatography confirmed at least two CBD stone with prominent CBD $\sim 6 \mathrm{~mm}$ (Fig. 1B). Laparoscopic cholecystectomy was planned, but prior that biliary clearance needs to be done. With altered surgical anatomy post RYGB, conventional ERCP was not possible; however, enteroscopy-assisted ERCP utilizing the biliopancreatic limb was still feasible, but technically challenging with low rate of successful biliary cannulation ( $60 \%$ only). Another disadvantage was need for a second procedure for laparoscopic cholecystectomy. CBD was nearly normal in size; hence, laparoscopic CBD exploration was not contemplated. Finally, a decision for LA-ERCP was made. Here, a temporary access is made through laparoscopic surgical gastrostomy through which a side view endoscope is passed into the remnant gastric pouch and then to the papilla just like conventional ERCP.

\section{Procedure Description}

The procedure was done in operating theater after prior informed consent explaining the procedures and related complications in detail. Perioperative prophylactic intravenous antibiotics was given and rectal indomethacin suppository (100 mg) was given for prophylaxis of post-ERCP pancreatitis. Proper positioning and draping were done under sterile conditions. Pneumoperitoneum was created through the standard $10 \mathrm{~mm}$ umbilical port using carbon dioxide up to a pressure of $12 \mathrm{~mm} \mathrm{Hg}$ with a Veress needle. A $10 \mathrm{~mm}$ trocar was placed in the epigastrium and two other $5 \mathrm{~mm}$ trocars were placed in the right and left flanks for surgical access. Another $15 \mathrm{~mm}$ trocar was placed in left hypochondrium that was used to visualize the gastric remnant (Fig. 2A). Gastrostomy was done at a site on the greater curvature of the stomach using cautery. Two stay sutures were placed through the anterior abdominal wall around the gastrostomy site to secure this position (Fig. 2B). ERCP scope was inserted through $15 \mathrm{~mm}$ left hypochondrium port and passed through the gastrostomy site into the remnant stomach under laparoscopic guidance (Fig. 2C). The scope was further advanced into the duodenum in the conventional manner. Papilla was identified (Fig. 2D). Wire-guided biliary cannulation was done. Cholangiogram showed mildly prominent CBD with a filling defect at the lower end (Fig. 2E). Sphincterotomy was done (Fig. 2F). Repeated balloon sweep extracted one yellowish stone (Fig. 2G, H). Check cholangiogram was normal. Following completion of ERCP, the scope was withdrawn and gastrostomy site was closed 

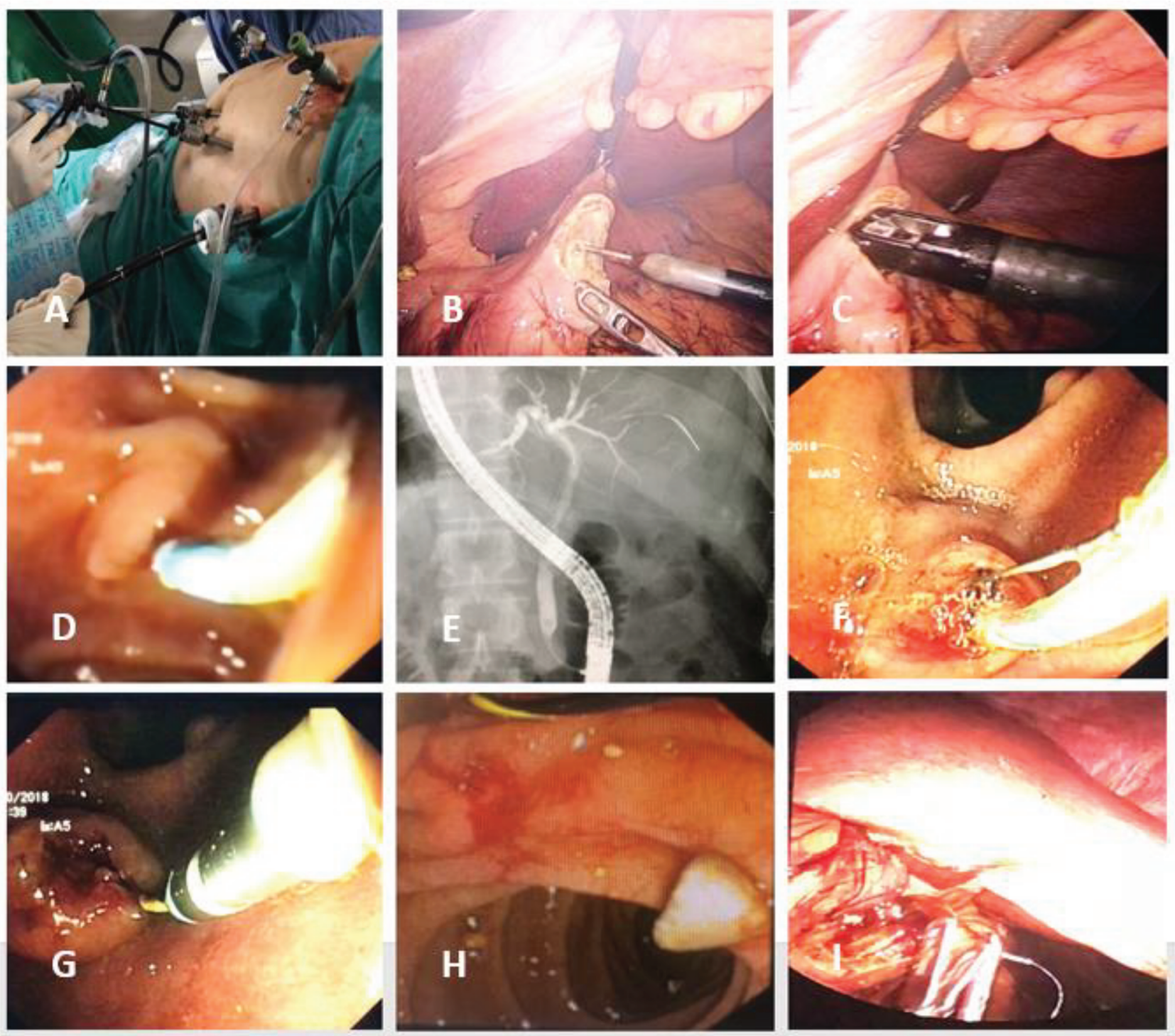

Fig. 2 Procedural steps in laparoscopy-assisted transgastric endoscopic retrograde cholangiopancreatography (LA-ERCP): (A) standard laparoscopy ports with an additional $15 \mathrm{~mm}$ port in left hypochondrium for insertion of ERCP scope, (B) formation of gastrostomy on the greater curvature of the gastric remnant, (C) insertion of ERCP scope inside the remnant gastric cavity through the gastrostomy site. Standard ERCP steps: (D) biliary cannulation, (E) cholangiogram showing common bile duct (CBD) stone, (F) sphincterotomy, (G) balloon sweep, (H) CBD stone extraction and biliary clearance, (I) laparoscopic closure of gastrostomy site with sutures and staplers.

with 2.0 Vicryl sutures followed by stapler using a $3.5 \mathrm{~mm}$ staple load (Fig. 2I). After biliary clearance, laparoscopic cholecystectomy was completed by standard technique in the same setting. The whole procedure was uneventful. He was kept admitted for one more day and discharged the following day. On follow-up after 4 weeks, he was absolutely asymptomatic with completely normal liver function test.

\section{Discussion}

Different bariatric surgery has evolved over time; RYGB is commonly performed surgery for weight loss. Rapid weight loss post-surgery is a known risk factor for gallstones and choledocholithiasis.

Cholelithiasis is common after gastric bypass surgery, and is seen in up to $40 \%$ of patients within 6 months of surgery ${ }^{2}$ and up to $40 \%$ of these become symptomatic and require cholecystectomy. ${ }^{1}$ Choledocholithiasis can occur years after both cholecystectomy and RYGB. Average time since RYGB in one series was 6.9 years and $43 \%$ of the patients presented 1 to 2 years after initial cholecystectomy. ${ }^{4} \mathrm{~A}$ retrospective review by Brockmeyer et al assessed the biliary complications of RYGB in 1,366 patients, of which 380 had cholecystectomy prior RYGB and only 3 of them $(<1 \%)$ developed primary CBD stones. This result suggests that RYGB itself can predispose to primary CBD stone, but it is a relatively rare event to occur. ${ }^{5}$ The more common scenario is presentation with both gallstones and CBD stone requiring biliary clearance and cholecystectomy at the same time. Altered surgical anatomy post RYGB makes endoscopic access to the second part of duodenum difficult and technically challenging. Options are as follows: 
a. BEA-ERCP-technical successful biliary cannulation $\sim 60 \%{ }^{6}$

b. Concomitant laparoscopic cholecystectomy with bile duct exploration.

c. Endoscopic ultrasound/fluoroscopy-guided gastrostomy tube insertion followed by a second-stage ERCP procedure through the gastrostomy tube. ${ }^{7}$

d. LA-ERCP-biliary cannulation rate $~ 90$ to $100 \%{ }^{8}$

BEA-ERCP for biliary clearance post-bariatric RYGB is practically feasible, but the chances of successful biliary cannulation with this modality even at best hands are not more than $60 \%$. With this surgical rearrangement, the total limb length, which is the sum total of Roux limb and pancreaticobiliary limb from the ligament of Treitz to the jejunojejunal anastomosis, is the deciding factor for BEA-ERCP. A standard gastric bypass usually involves a total limb length of at least $150 \mathrm{~cm} .{ }^{9}$ Those with total limb length less than $150 \mathrm{~cm}$ are reported to have higher therapeutic success compared with those with total limb length of $150 \mathrm{~cm}$ or more (88 vs. $25 \%$, respectively). ${ }^{1}$ The main disadvantage of this procedure is long procedure time as it first involves identification of enteroenterostomy site that is often time consuming and difficult orientation of papilla for cannulation. The papillary access is not only difficult, but the angle at which the papilla is reached is often too awkward for cannulation. Conventional side view endoscope cannot be used and enteroscopes do not have elevators to hold the accessories; hence, precise scope positioning for cannulation is difficult. Another disadvantage of BAE-ERCP is a need for a second procedure for cholecystectomy and often at different setting. Schapira et al first reported his technique of endoscopy using the preexisting open gastrostomies for both gastrointestinal bleeding and ERCP in $1975 .{ }^{10}$ Peters et al first described laparoscopic gastrostomy done specifically for ERCP (LA-ERCP) in 2002. ${ }^{11}$ In 2007, Ceppa et al further reported simplified approach in 10 patients using the purse-string technique to fix the gastrostomy site and immediate post-procedure gastrotomy closure with sutures or staples. LA-ERCP has several advantages over BAE-ERCP; ERCP can be performed using conventional side-view endoscope; both ERCP and laparoscopic cholecystectomy can be done consecutively as a single procedure. The published data suggest higher rate of papillary identification, successful biliary cannulation (90-100\%), higher therapeutic success with low associated complication rate $(<5 \%)$ with LA-ERCP when compared with BEA-ERCP. ${ }^{12,13}$ Even this technique can be challenging at times, transgastric access in LA-ERCP with previous abdominal surgery, multiple laparoscopic interventions, history of peritonitis, or abdominal abscess is sometimes difficult due to intense postoperative adhesions. Since only a small portion of the scope is inside the gastrostomy site $(20 \mathrm{~cm})$, the scope stability for negotiation through the pylorus into the second part of the duodenum and proper orientation for cannulation is not easy and needs good expertise. Planning and organizing LA-ERCP is another challenge since it has to be done in operation theater and all the endoscopic accessories, scopes, and electrocautery units need to be shifted and properly placed. The mobile " $\mathrm{C}$ " arm is often used for fluoroscopy, the quality of which is not comparable to that in ERCP room. Another major problem with LA-ERCP is when there is a need for biliary stents due to incomplete biliary clearance or prophylactic pancreatic stent with repeated inadvertent pancreatic duct entry or post-sphincterotomy bleeds that may need an additional endoscopic procedure to either remove stents, clear residual stones, or control bleeding. In such cases, repeat LA-ERCP may be required that adds on to additional procedure, costs, and complications. Hence, the preoperative assessment of CBD stones is essential and procedure should be contemplated only if the chances of complete biliary clearance are most likely and in cases where the prophylactic pancreatic duct stent is required, a $3 \mathrm{~F}$ stent should be placed that is expected to migrate spontaneously in $\sim 85 \%$ cases. ${ }^{14}$ Moreover, when the need for repeat ERCP is expected during the first LA-ERCP, recommendation is to place a gastrostomy tube through the gastrotomy site to complete subsequent interventions as required. The gastrotomy site on the greater curvature should be made as lateral as possible and often a laparoscopic trocar of 15 or $18 \mathrm{~mm}$ is used to guide the smooth passage of scope through the pylorus. ${ }^{15}$ The gastrostomy site should be tightly fixed with purse string sutures to prevent losing the insufflation pressure. Insufflation is mostly done with carbon dioxide, which would easily get absorbed in cases with intraperitoneal leakage; at the same time bowel distension due to excess gas causes difficulty in completing the following cholecystectomy procedure. This could be prevented by application of clamp proximal to the ligament of Treitz. Biliary cannulation can sometimes be difficult despite all efforts; in such scenario laparoscopic access to cystic duct renders an additional advantage for retrograde wire insertion to complete ERCP by rendezvous technique. This technique of LA-ERCP can also be utilized to treat the cystic stump leak.

\section{Conclusion}

A subset of patients may develop gallstone disease with choledocholithiasis after RYGB that needs biliary clearance. LA-ERCP by transgastric access has been shown to be safe and effective technique to gain access to the biliary system post RYGB and a much superior approach than BEA-ERCP as cholecystectomy can be done in the same session avoiding the need for a second procedure. Hence, post-bariatric RYGB with a complicated gallstone disease like choledocholithiasis, cholangitis, or any other biliopancreatic disorder combining cholecystectomy and LA-ERCP as a first-line approach is a valid treatment strategy with equivalent morbidity, same duration of hospital stay, and reduced total costs.
Video 1
Procedural steps of Laproscopic assisted transgastric ERCP (LA-ERCP)
Online content including video sequences viewable at: https://www.thieme-connect.com/products/ejournals/ html/10.1055/s-0041-1728221 


\section{Conflict of Interest}

None.

\section{References}

1 Shiffman ML, Sugerman HJ, Kellum JM, Brewer WH, Moore EW. Gallstone formation after rapid weight loss: a prospective study in patients undergoing gastric bypass surgery for treatment of morbid obesity. Am J Gastroenterol 1991;86(8):1000-1005

2 Deitel M, Petrov I. Incidence of symptomatic gallstones after bariatric operations. Surg Gynecol Obstet 1987;164(6):549-552

3 Weiss AC, Inui T, Parina R, et al. Concomitant cholecystectomy should be routinely performed with laparoscopic Roux-en-Y gastric bypass. Surg Endosc 2014

4 Habenicht Yancey K, McCormack LK, McNatt SS, Powell MS, Fernandez AZ, Westcott CJ. Laparoscopic-assisted transgastric ERCP: a single-institution experience. J Obes 2018;2018:8275 96510.1155/2018/8275965

5 Brockmeyer JR, Grover BT, Kallies KJ, Kothari SN. Management of biliary symptoms after bariatric surgery. AmJSurg 2015;210(6): 1010-1016, discussion 1016-1017

6 Schreiner MA, Chang L, Gluck M, et al. Laparoscopy-assisted versus balloon enteroscopy-assisted ERCP in bariatric post-Rouxen-Y gastric bypass patients. Gastrointest Endosc 2012;75(4): 748-756

7 Attam R, Leslie D, Freeman M, Ikramuddin S, Andrade R. EUSassisted, fluoroscopically guided gastrostomy tube placement in patients with Roux-en-Y gastric bypass: a novel technique for access to the gastric remnant. Gastrointest Endosc 2011; 74(3):677-682

8 Lopes TL, Clements RH, Wilcox CM. Laparoscopy-assisted ERCP: experience of a high-volume bariatric surgery center (with video) Gastrointest Endosc 2009;70(6):1254-1259

9 Sarhan M, Choi JJ, Al Sawwaf M, Murtaza G, Getty JL, Ahmed L. Is weight loss better sustained with long-limb gastric bypass in the super-obese? Obes Surg 2011;21(9):1337-1343

10 Schapira L, Falkenstein DB, Zimmon DS. Endoscopy and retrograde cholangiography via gastrostomy. Gastrointest Endosc 1975;22(2):103

11 Peters M, Papasavas PK, Caushaj PF, Kania RJ, Gagné DJ. Laparoscopic transgastric endoscopic retrograde cholangiopancreatography for benign common bile duct stricture after Roux-en-Y gastric bypass. Surg Endosc 2002;16(7):1106

12 Ceppa FA, Gagné DJ, Papasavas PK, Caushaj PF. Laparoscopic transgastric endoscopy after Roux-en-Y gastric bypass. Surg Obes Relat Dis 2007;3(1):21-24

13 Nguyen NT, Hinojosa MW, Slone J, Lee J, Khiatani V, Wilson SE. Laparoscopic transgastric access to the biliary tree after Rouxen-Y gastric bypass. Obes Surg 2007;17(3):416-419

14 Sofuni A, Maguchi H, Itoi T, et al. Prophylaxis of post-endoscopic retrograde cholangiopancreatography pancreatitis by an endoscopic pancreatic spontaneous dislodgement stent. Clin Gastroenterol Hepatol 2007;5(11):1339-1346

15 Geert P, Jacques H, Guido L. ERCP by laparoscopic transgastric access and cholecystectomy at the same time in a patient with gastric bypass who was seen with choledocholithiasis. Gastrointest Endosc 2010;72(5):1115-1116 